\title{
Forstpolitische und forstrechtliche Probleme von aufgegebenem Kulturland
}

\begin{abstract}
Vorbemerkungen
Seit der grundlegenden Arbeit von SURBER (1973) ist der Begriff «Brachland» in zahlreichen Publikationen verwendet und immer vieldeutiger geworden. Die von SCHERRER (1980) aufgelisteten Definitionen lassen vermuten, daß die verschiedenen Aspekte des Brachlandes sich nicht in einer einzigen Beschreibung fassen lassen. Im vorliegenden Beitrag wird der Begriff Brachland möglichst vermieden und durch «aufgegebenes Kulturland» ersetzt. Denn aus forstpolitischer und forstrechtlicher Sicht ist von ausschlaggebender Bedeutung, daß die Landwirtschaft in den Problemgebieten stetig zurückgeht, d.h. in immer bedeutenderem Ausmaß extensiviert wird. Ohne menschliches Dazutun wird der größte Teil des aufgegebenen Kulturlandes über kurz oder lang zu Wald. Zu diesem Zeitpunkt hat sich der Forstdienst von Gesetzes wegen mit den einwachsenden Flächen zu befassen. Diese Vegetationsfolge veranlaßte die forstliche Forschung schon vor längerer Zeit, sich dem Brachlandproblem anzunehmen. Vorerst wurde versucht, das Ausma $ß$ und die Bedeutung der nicht mehr genutzten Landwirtschaftsflächen abzuschätzen. Mit unseren laufenden Forschungsprojekten über die Behandlung von aufgegebenem Kulturland wird beabsichtigt, zur interdisziplinären Erarbeitung integraler Planungsmethoden beizutragen.
\end{abstract}

\section{Forstpolitische Aspekte}

\subsection{Waldflächenzunahme}

Das im Bundesgesetz betreffend die eidgenössische Oberaufsicht über die Forstpolizei (vom 11.Oktober 1902) in Art. 31 verankerte Walderhaltungsgebot ist und bleibt Eckpfeiler der schweizerischen Forstpolitik. Hinsichtlich der Waldvermehrung hält das Bundesgesetz in Art. 3 und Art. 36 fest: Es ist darauf hinzuwirken, daß unbewaldete Grundstücke zur Waldbestockung gelangen (Aufforstung oder natürliche Wiederbewaldung), wenn damit Schutzwaldungen im Einzugsgebiet von Wildwassern, Lawinen, Rutschungen usw. begründet werden können. Mit der Unterstützung von Bundes- und Kantonssubventionen wurden im schweizerischen Berggebiet gegen
38000 ha Wald aufgeforstet. Die bisher natürlich eingewachsenen Flächen leisten in den meisten Gebieten ebenfalls wichtige Schutzfunktionen und sind deshalb im allgemeinen erwünscht.

Die Waldvermehrung im Gebirge wird künftig noch große Anstrengungen erfordern. So stellen WICKI und WEBER (1980) fest, daß in den Entwicklungskonzepten von 35 untersuchten Bergregionen große Aufforstungsvorhaben erwähnt werden. Die zunehmende touristische Nutzung und immer dichtere Verkehrsnetze verlangen in vielen Gebieten erhöhten Schutz vor den Naturgefahren. Dabei decken sich die Interessen von Nutznießern der Waldwirkungen oft nicht mit den Absichten der Grundeigentümer. Eine Aufforstung verlangt während langer Zeit beträchtliche Investitionen in Pflanzungen, Pflege- und Schutzmaßnahmen. Fast alle Berggebiete der Schweiz weisen so hohe Wildbestände auf, daß die Projekte beträchtlich verteuert werden. Der Aufforstungserfolg ist vielerorts sogar in Frage gestellt. Die Grundeigentümer sind deshalb an Aufforstungen oder an gezielter waldbaulicher Pflege des sich natürlich bewaldenden Kulturlandes kaum interessiert. Landwirtschaftlich nicht mehr genutzte Flächen liegen häufig in Gebieten mit stark parzelliertem Privateigentum. Der Einwuchs oder die Aufforstung führen zu einzelnen kleinen Waldparzellen, die später auch forstlich nicht kostendeckend bewirtschaftet werden können.

Soll die Öffentlichkeit nun grundsätzlich aufgegebene Kulturflächen aufkaufen und wiederbewalden? Überlegungen zu dieser Frage müssen davon ausgehen, da $ß$ nicht jede Waldvermehrung a priori von Vorteil ist. Wachsen größere Gebiete ein, so verändert sich beispielsweise der Gesamtaspekt einer Landschaft. Der wertvolle Wechsel zwischen Wald und offener Flur geht verloren. Möglicherweise werden auch seltene Biotope zerstört, deren Erhaltung höher einzuschätzen ist als ein Waldflächengewinn. Die Bewaldung von offenem Ski- und Abfahrtsgelände kann zu Konflikten mit touristischen Interessen führen. Schließlich können neue Waldflächen die angrenzenden landwirtschaftlich noch genutzten Flächen beschatten und deren Bewirtschaftung erschweren.

Dr. Fritz Pfister, Eidg. Anstalt für das forstliche Versuchswesen, 8903 Birmensdorf 
In der Gesamtkonzeption für eine schweizerische Wald- und Holzwirtschaftspolitik (Eidg. Departement des Innern, 1975) wird zur Vermeidung solcher Konflikte eine klare Vorstellung darüber gefordert, was in den verschiedenen Regionen mit dem aufgegebenen Kulturland $\mathrm{zu}$ geschehen hat. In diesem Zusammenhang wird auch die Bewilligungspflicht für Neuaufforstungen vorgeschlagen. Was aber kann unternommen werden, wenn bei einer Verweigerung der Aufforstungsbewilligung ein Eigentümer sich nicht mehr um seine Parzelle kümmert und diese einwachsen läßt? Wie Bosshard (1975) deutlich macht, enthält die heute gültige Gesetzgebung keine Mittel, um einen Grundeigentümer daran zu hindern, seinen Boden dem Wald zurückzugeben. Die Zukunft wird zeigen, ob gestützt auf die Raumplanungsgesetze eine minimale Bewirtschaftung gefordert werden kann. Früher oder später müssen die Probleme gelöst werden, die entstehen, wenn sich den Bau-, Landwirtschafts- und Schutzzonen gewidmete Flächen ohne Zutun des Grundeigentümers und gegen das allgemeine Interesse wiederbewalden. Vorläufig geben die bodenrechtlich liberalen Verhältnisse der Schweiz den Planern und Forstleuten nur sehr beschränkte Möglichkeiten, die Waldflächenentwicklung zu beeinflussen.

\subsection{Mögliche Nutzungen von aufgegebenen Kulturflächen}

Es existiert eine umfangreiche Literatur über verschiedene Möglichkeiten, aufgegebenes Kulturland zu nutzen (z.B. SCHERRER und SURBER, 1978). Die Abbildung 1 macht deutlich, da $\beta$ Wald nur eine von vielen möglichen Folgenutzungen ist. Bevor man sich zur forstlichen Bewirtschaftung entschließt, sollten die anderen Nutzungsformen sorgfältig abgeklärt werden. Denn eine Bewaldung ist praktisch irreversibel, unabhängig davon, ob sie absichtlich oder zufällig entstanden ist. Dies kommt auch in den forstrechtlichen Bestimmungen zum Ausdruck.

Solange sich keine anderen Interessen für das aufgegebene Kulturland zeigen und solange das Stadium der Vegetationsentwicklung die Unterstellung unter das Forstgesetz noch nicht verlangt, ist der Planungsund Entwicklungsspielraum noch viel offener. Wenn der Eigentümer die Einwüchse periodisch zurückschneidet, kann er sich diese Freiheiten über lange Zeit erhalten. Mit Blick auf die unsicheren Prognosen über den künftigen Bedarf an Landwirtschaftsflächen ist es angezeigt, geeignete Kulturflächen mit minimalem Pflegeaufwand offenzuhalten. Eine Störung der Landesversorgung mit Lebensmitteln kann die wirtschaftliche Lage der Landwirtschaft plötzlich ändern. In diesem Fall ist nicht auszuschließen, daß eine große Zahl von Gesuchen zur Rodung wiederbewaldeter Flächen gestellt wird. Als Folge einer derartigen Entwicklung entstünden hohe, durch eine rechtzeitige
Planung vermeidbare Rekultivierungskosten. Im weiteren müßte eine weitgehende Gefährdung des Walderhaltungsgebotes befürchtet werden.

Ein Entscheid zur Wiederbewaldung muß auch aus der Sicht der Bewirtschaftung der Wälder gut überlegt werden. Die Waldbauer des In- und Auslandes sind sich darüber einig, da $B$ verschiedene Dienstleistungen des Waldes nur gewährleistet sind, wenn Pflegeeingriffe regelmäßig durchgeführt werden. Untersuchungen des Schweizer Waldes haben nun aber gezeigt, da $ß$ im Berggebiet ca. 100000 ha nur in unregelmäßigem Turnus und ca. 70000 ha in den nächsten 30 Jahren voraussichtlich überhaupt nicht genutzt und gepflegt werden. Die Bewirtschaftungsprobleme in der Forstwirtschaft erstrecken sich also heute schon über Flächen in der Größenordnung des von der Landwirtschaft aufgegebenen Kulturlandes. Aus diesem Grunde muß angenommen werden, $\mathrm{da} B$ auch die zuwachsenden Waldflächen nicht rechtzeitig behandelt werden können. Ohne gezielte waldbauliche Eingriffe ist aber die natürlich entstandene Bestokkung meistens wenig wertvoll. Dies sowohl im Hinblick auf die Holzproduktion als auch auf die Dienstleistungen des Waldes.

\section{Forstrechtliche Aspekte}

\subsection{Waldbegriff}

Zur Entstehungszeit des Forstgesetzes wurde es nicht als nötig erachtet, den Waldbegriff gesetzlich zu verankern. Erst die immer intensivere Bodennutzung in den Agglomerationsgebieten des Mittellandes, aber auch die zunehmende Extensivierung der Landwirtschaft und "Verwaldung» im Berggebiet forderten eine Umschreibung des Waldes. Das Bedürfnis nach mehr Rechtssicherheit führte 1965 zur bundesrechtlichen Begriffsbestimmung von Wald in Art. 1 der Vollziehungsverordnung zum Forstpolizeigesetz. Was rechtlich Wald ist, bestimmen danach die tatsächlichen Verhältnisse. Das Forstrecht geht nicht von einem in Plänen festgelegten oder in Absichten und Erlassen fixierten Waldareal aus. Die Walddefinition hält fest, $\mathrm{da} B$ ungeachtet der Entstehung, Nutzungsart oder Bezeichnung im Grundbuch eine Fläche dann als Wald gilt, wenn sie mit Waldbäumen bestockt ist, Holz erzeugt oder geeignet ist, Schutz- und Wohlfahrtswirkungen auszuüben.

Liegt Wald im Sinne dieser Definition vor, ist eine Umwandlung in Nichtwald nur über eine Rodungsbewilligung möglich. Die Unterstellung einer Parzelle unter das Forstgesetz engt den Grundbesitzer in seiner Eigentumsfreiheit stark ein. Für die Waldbewirtschaftung gelten zudem besondere Vorschriften wie das Kahlschlagsverbot, das Nachhaltigkeitsgebot und das Verbot von schädlichen Nebennutzungen. Es gibt deshalb immer wieder Streitfälle, in denen Eigentümer von weitgehend bestockten Flächen die 


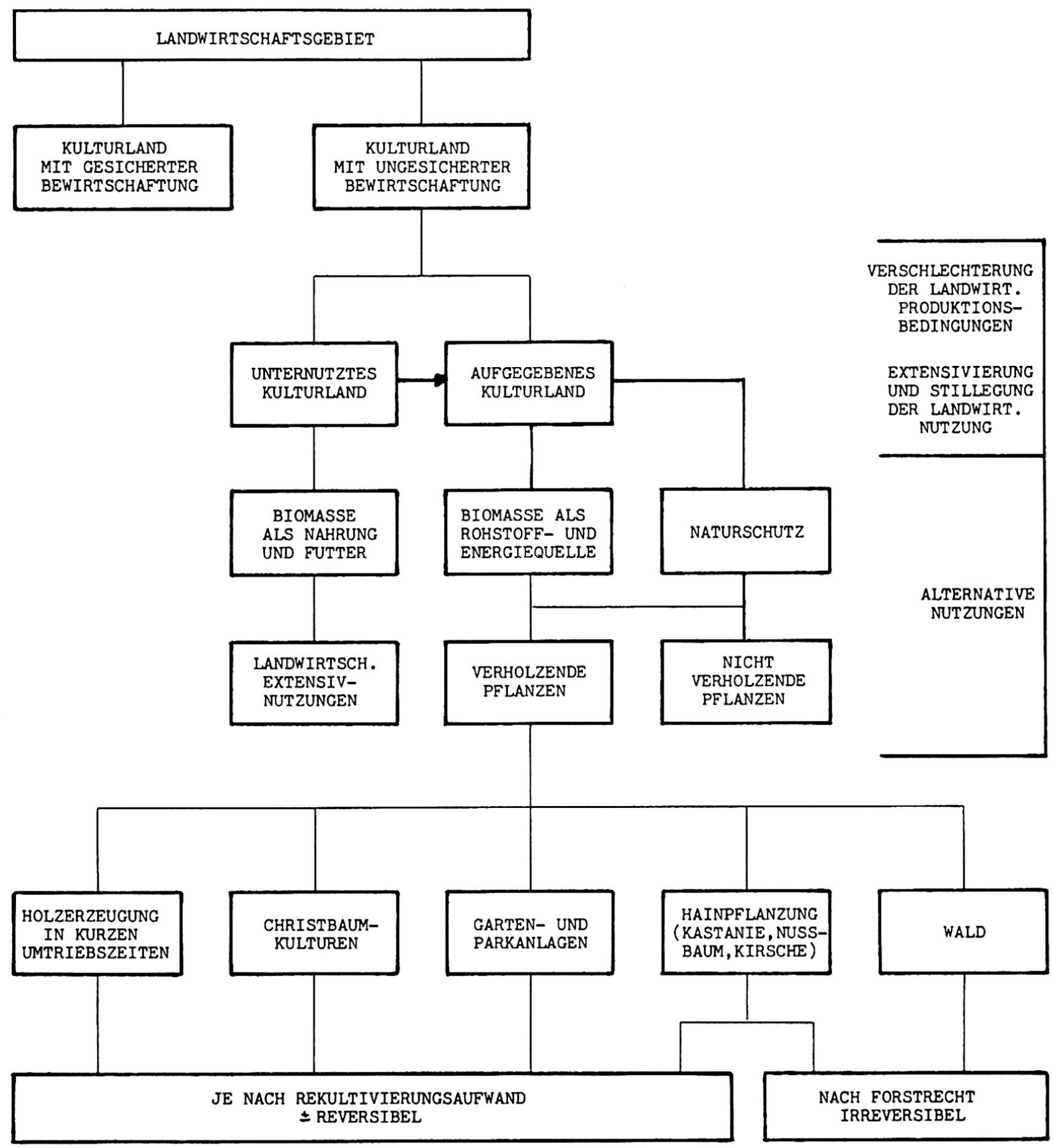

Abb. 1 Wald ist nur eine von vielen möglichen Nutzungen von aufgegebenem Kulturland

Waldqualifikation abzuweisen versuchen. Gestützt auf die Praxis des Bundesgerichts zu Rechtsfragen der Walderhaltung hat DUBS (1974) darauf hingewiesen, $\mathrm{da} ß$ dem Prinzip der Walderhaltung auch der neu entstandene oder neu angelegte Wald untersteht. Sobald eine Baumbestockung auf einer landwirtschaftlich nicht mehr genutzten Fläche im Rechtssinn als
Wald bezeichnet werden muß, ist das Gebot zu seiner Erhaltung unbestritten. Der eingewachsene Jungwald stellt ein wertvolles Nutzungspotential dar, das nur bei überwiegenden Interessen und nach sorgfältiger Abwägung wieder vernichtet werden sollte. Die restriktive Bewilligungspraxis von Rodungsgesuchen hat auch eine gewisse wirtschaftliche Berechtigung. 
Mit der Walderhaltung soll nicht nur die Fläche insgesamt, sondern auch die räumliche Verteilung bewahrt werden (Art. 26 der Vollziehungsverordnung zum Bundesgesetz über die Forstpolizei). Die Meinung, da $B$ Waldvermehrungen im Berggebiet als Ersatz für Rodungen im Mittelland betrachtet werden können, hält vor dem Gesetz nicht stand.

\subsection{Wann werden einwachsende Flächen zu Wald?}

Das Forstrecht sieht vor, daß Flächen besonders dann aufgeforstet und gegebenenfalls durch technische Maßnahmen geschützt werden sollen, wenn damit der Schutz vor Naturgefahren erhöht werden kann. Oft fehlen aber großräumige Vorstellungen darüber, welche Flächen aufgeforstet oder durch natürlichen Einwuchs bewaldet werden sollen. Das Bundesgesetz über Bewirtschaftungsbeiträge an die Landwirtschaft mit erschwerten Produktionsbedingungen (vom 14. Dezember 1979) bezweckt, möglichst viele geeignete Flächen der landwirtschaftlichen Nutzung zu erhalten. Wenn die zum Schutz vor Naturgefahren forstlich zu nutzenden Flächen nicht rechtzeitig ausgeschieden werden, kann dies in Extremfällen dazu führen, daß mit landwirtschaftlichen Subventionen eine Bewaldung verhindert wird, wo mit forstlichen Beiträgen eine Wiederbewaldung gefördert werden müßte.

Abb. 2 Verwaldungsprozess in einem Gebiet der Güterzusammenlegung Sonvico, Kanton Tessin

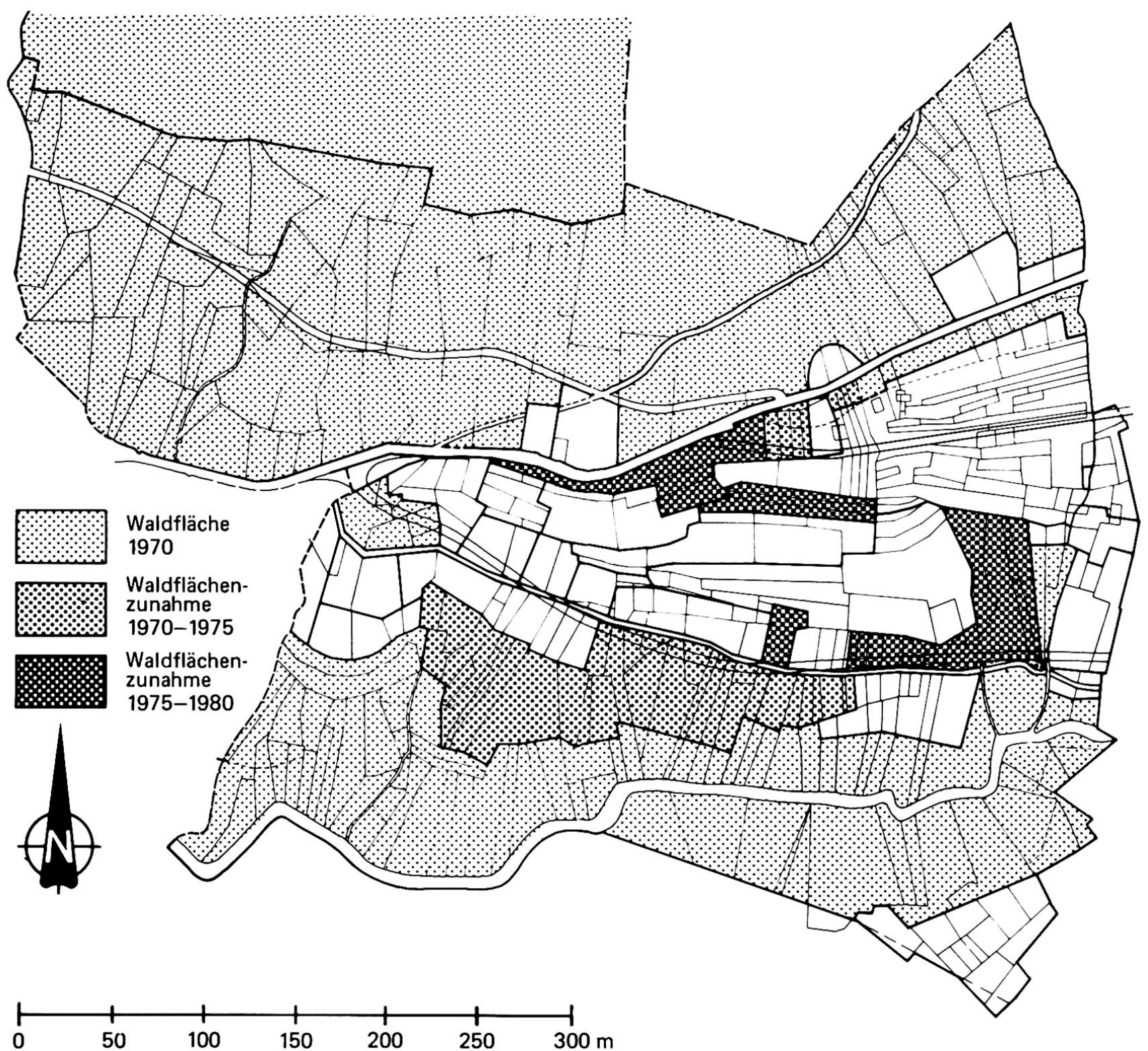


Auch aus Gründen der Rechtssicherheit sollte eine gezielte Wiederbewaldungs- und Aufforstungspolitik die Entstehung neuer Waldflächen frühzeitig festlegen. Das Walderhaltungsgebot will den Eigentümer nicht grundsätzlich daran hindern, die in sein Land eindringende Waldvegetation abzuwehren. Es soll auch nicht alles einwachsende Land irreversibel dem Wald zugeschlagen werden. Der vorhandene und aus Schutzinteressen erwünschte «neue» Wald soll erhalten bleiben.

Die bundesrechtliche Definition ist eine Rahmenbestimmung. Den Kantonen ist ein gewisser Spielraum überlassen, in diesem Rahmen den Waldbegriff zu konkretisieren. Besonders zu den Beurteilungselementen Dichte, Alter und Fläche haben einzelne Kantone Vorschriften erlassen. Zürich und Aargau legen beispielsweise ein Mindestalter von 10 Jahren fest. Der Kanton Graubünden hat kürzlich eine Richtlinie erlassen, nach der eine bestockte Fläche als Wald gilt, wenn der Beschirmungsgrad über 0.3 liegt, die Mehrheit der Bestandesglieder mindestens 15 Jahre alt und $2 \mathrm{~m}$ hoch oder mindestens 10 Jahre alt und $7 \mathrm{~m}$ hoch sind. Im Bundesgerichtsentscheid zum Fall «Nürensdorf», Kanton Zürich (BGE 107 Ib 50), wurde 1981 festgehalten, daß Normen und Richtlinien den Besonderheiten Rechnung tragen müssen, weil sonst einem Schematismus Vorschub geleistet würde, der dem Forstpolizeirecht sonst fremd ist. Dementsprechend hat das Bundesgericht verschiedentlich die Auffassung vertreten, daß die Altersgrenze nicht starr auf 10 Jahre fixiert werden soll.

Die Frage, wann eine einwachsende Fläche zu Wald wird, erlangt besonders im Kanton Tessin zunehmende Bedeutung. Hier stellt der Verwaldungsproze $B$ oft schwerwiegende Probleme. Ausgedehnte Flächen werden von der Landwirtschaft aufgegeben und bewalden sich unter klimatisch günstigen Bedingungen in kurzer Zeit. Die Abbildung 2 zeigt einen Ausschnitt des Güterzusammenlegungsplanes von Sonvico. Während der Durchführung dieses Projektes mußte das Waldareal dreimal neu bestimmt werden. Bereits fünf Jahre nach der Erhebung der Eigentumsverhältnisse waren im abgebildeten Ausschnitt rund 1,3 ha eingewachsen. Bis zur letzten Kartierung, 10 Jahre nach Beginn der Arbeiten, hat sich der Wald nochmals um fast 1 ha vergrößert. Wenn die Bewaldung wie bisher fortschreitet, so werden auch die ertragreicheren offenen Flächen trotz der Melioration bald aufgegeben.

Das Bundesgericht behandelte die Problematik von einwachsenden Flächen erstmals im Jahre 1972 im Tessiner Entscheid "Gilardi-Morcote» (BGE 98 Ib 364). Es strich in seinen Begründungen die Bedeutung der Frage hervor, ob der Eigentümer sich bemüht hat, sein Land vor einer ungewollten Umwandlung in Wald zu bewahren. Die Maßnahmen des Eigentümers sind unter Berücksichtigung der jeweiligen Umstände zu würdigen. Hat er das zur Verhinderung der Bewaldung getan, was ihm vernünftigerweise zugemutet werden kann, so wird der Waldcharakter verneint, auch wenn die Bestockung älter als $10 \mathrm{Jahre}$ ist. Dies heißt, daß umgekehrt nach 10-15 Jahren Wald im Rechtssinne entstehen kann, sofern gegen das Einwachsen von aufgegebenem Kulturland nichts unternommen wird. Im Fall «Küsnacht), Kanton Zürich (BGE 107 Ib 355), hat das Bundesgericht diese Auffassung bestätigt. Es unterscheidet in seinen Urteilserwägungen zwischen Tat- und Rechtsfragen. In tatsächlicher Hinsicht sind der vorhandene Wuchs (Dichte, Alter, Ausmaß) sowie der Zusammenhang mit benachbarten Waldungen zu beurteilen. Zudem ist von Bedeutung, ob die Fläche Schutz- und Wohlfahrtswirkungen auszuüben vermag. Rechtsfrage ist, wie der Begriff des Waldes und dessen einzelne Elemente ausgelegt werden. Zum Alter wird erwogen: «Ist der Wuchs jedoch älter als 10-15 Jahre, so nimmt die bundesgerichtliche Rechtssprechung auch gegen den Willen des Eigentümers das Vorhandensein von Wald im Rechtssinne an.»

\section{Künftige Forschung aus forstlicher Sicht}

\subsection{Waldkataster}

Die Gerichtspraxis stellt bei der Beurteilung der Waldqualifikation, wie wir gesehen haben, auch auf das Alter der Bestockung ab. Ein Waldkataster vermag die Rechtssicherheit deshalb wesentlich zu verbessern. Man kann sich die Behandlung von Einwüchsen mit diesem Instrument so vorstellen, da $B$ das aufgegebene Kulturland z. B. im Rahmen der Erstellung oder Revision von Richt- und Nutzungsplänen periodisch erfaßt wird. Eigentümer von weitgehend bewaldeten Parzellen werden darauf aufmerksam gemacht, da $\beta$ nicht zurückgeschnittene Flächen nach einer angemessenen Frist in den Waldkataster aufgenommen werden. Der Umstand, daß die forstrechtliche Definition des Waldareals sich der tatsächlichen Entwicklung anpaßt, ist nach BLOETZER (1975) für die periodische Ausscheidung des Waldes kein unüberwindbares Hindernis.

Im Zusammenhang mit der zweckmäßigen Erfassung des Waldareals hat die Forschung folgende Aufgaben: Entwicklung und Testen von verschiedenen Kartierungsverfahren zur Feststellung wichtiger Vegetationsentwicklungsstadien im aufgegebenen Kulturland. Besonders die Übergangsphase von der starken Verbuschung zum Wald muß rechtzeitig erhoben werden.

Die Erstellung und Nachführung eines Waldkatasters sind auf die Genauigkeitsanforderungen abzustimmen. Je nach der Bedeutung der Waldabgrenzung genügen grobe Übersichtskarten oder sind genaue Waldfeststellungen erforderlich. Gegenüber dem Landwirtschaftsgebiet geben Karten im Maßstab 1:5000 oder 1:10000 meistens ausreichende Informa- 
tionen. Die Abgrenzung gegenüber dem Siedlungsgebiet verlangt dagegen eine feinere und genauere Erfassung des Waldrandes in Karten im Maßstab $1: 1000$ oder $1: 2000$.

Es muß allerdings deutlich darauf hingewiesen werden, da $\beta$ der Waldkataster ein verwaltungsinternes Instrument mit beschränkter Verbindlichkeit ist. Im strittigen Einzelfall gilt nach wie vor die Waldfeststellung, die durch den Forstdienst nach dem bundesrechtlichen Waldbegriff und den kantonalen Zusatzbestimmungen vorzunehmen ist.

\subsection{Wiederbewaldungsplan}

Eine gezielte Ausscheidung der Flächen, die einer forstlichen Nutzung zugeführt werden sollen, fordert Untersuchungen des Bedeutungswandels (Grundwasserschutz, Immissionen, Stabilität des Geländes usw.) der Wald-Schutzfunktionen. Unerwünschte Entwicklungen und Konflikte durch eine natürliche, nicht gesteuerte Bewaldung müssen vermieden werden. $\mathrm{Zu}$ diesem Zweck sollte die Forschung nach besseren Entscheidungsgrundlagen zum Nutzungswechsel in den verschiedenen Phasen der Vegetationsentwicklung suchen.

Die Gefahrenkarte ist eine der wichtigsten Grundlagen für die Wiederbewaldungsplanung. Verschiedene Erhebungsmethoden zur Lokalisierung instabiler oder unzweckmäßig genutzter Flächen sind zu testen und zu verbessern.

\subsection{Integralprojekte}

Ein Wiederbewaldungsplan steht in engem Zusammenhang mit der Ausscheidung von Landwirtschafts-, Naturschutz-, Bauzonen usw. Auch die verschiedenen Förderungsmaßnahmen des Bundes und der Kantone (z. B. Alpmeliorationen, Erschließungen, forsttechnische Maßnahmen) müssen in ihren Beziehungen zur Waldbegründung berücksichtigt werden. Schließlich sind die Ursachen und absehbaren Entwicklungen der Kulturlandaufgabe unter den gegebenen naturräumlichen Verhältnissen sowie sozialen und ökonomischen Gegebenheiten zu betrachten. Seit ca. 15 Jahren befassen sich immer mehr Forschungsstellen des In- und Auslandes mit einzelnen Aspekten der geschilderten Probleme. Künftige Untersuchungen sollten aber von der gesamtheitlichen Betrachtung der konkreten Probleme ausgehen, um daraus Folgerungen zur Durchsetzung von Maßnahmen abzuleiten.

Die integralen Sanierungsprojekte im Kanton Obwalden (Sarnersee-Wildbäche), Kanton Schwyz (Wägital), Kanton Freiburg (Sense) und im Fürstentum Liechtenstein (Malbun) zeigen deutlich, daß die vielschichtigen Zusammenhänge im Problemkomplex Brachland nur in einer umfassenden, interdisziplinären
Betrachtung richtig erkannt werden können. Die Forschung muß deshalb in erster Linie Methoden entwickeln, die rasch einen hinreichend genauen Überblick über problematische Veränderungen der Bewirtschaftung geben. Die Koordination der Maßnahmen ist unumgänglich, wenn die verschiedenen Förderungsbemühungen mit sparsamem Mitteleinsatz langfristig zu wirksamen Lösungen führen sollen.

\section{Zusammenfassung}

Das Forstgesetz und die Förderungsmaßnahmen des Bundes zielen darauf $a b$, Waldungen mit ausgesprochener Schutzfunktion zu vermehren. Die Wiederbewaldung der bisher natürlich eingewachsenen Flächen im Berggebiet ist aus forstpolitischer Sicht meistens erwünscht. Eine ungelenkte, großflächige Waldvermehrung, wie sie sich heute in manchen Gebirgsregionen abzeichnet, kann aber den Interessen der Allgemeinheit zuwiderlaufen.

Wald stellt nur eine von vielen möglichen Folgenutzungen des aufgegebenen Kulturlandes dar. Ein Entscheid zur forstlichen Nutzung muß sorgfältig und rechtzeitig überlegt werden, denn einwachsende Flächen gelten als Wald, sobald ihr Zustand die Bedingungen des gesetzlich verankerten Waldbegriffes erfüllt.

Das Walderhaltungsgebot will nicht grundsätzlich verhindern, daß der Eigentümer die ins offene Kulturland einwachsende Waldvegetation zurückschneidet. Unternimmt er aber nichts, um sein Land vor einer sich natürlich einstellenden Bestockung zu bewahren, so kann nach einem bestimmten Zeitraum Wald im Rechtssinn entstehen. Besondere Probleme stellen sich diesbezüglich im Kanton Tessin, wo die aufgegebenen Flächen unter den klimatisch günstigen Bedingungen in kurzer Zeit einwachsen.

Die künftige Forschung zu Problemen des Brachlandes sollte aus forstlicher Sicht auf die Entwicklung von praxistauglichen Methoden zur Erstellung und Nachführung eines Waldkatasters ausgerichtet werden. Zudem müssen Kartierungsverfahren und Kriterien zur Beurteilung von Waldfunktionen gesucht werden, mit denen die der Forstwirtschaft zuzuführenden Flächen in einem Wiederbewaldungsplan festgelegt werden können. Künftige Forschungsarbeiten sollten aber vor allem auch von gesamtheitlichen Betrachtungen und integralen Lösungsansätzen ausgehen. 


\section{Summary \\ Political and legal aspects of unutilised land from the point of view of forestry}

Federal forest statutes and measures for the promotion of forest aim at increasing forest stands with mainly protective function. From this viewpoint, the regeneration of the forest in the mountain regions is desirable. On the other hand, the uncontrolled spreading of forest vegetation over large areas, as already occurring in some mountain regions, can run contrary to public interests.

Forest is only one of the various forms of land-use for areas no longer cultivated. The question of reforestation of such land must be considered carefully; moreover, decisions must be taken in good time, since areas of natural forest regeneration legally become forest as soon as they correspond to the definition set out by the law.

\section{Literaturverzeichnis}

BLOETZER, G. (1975): Forstliche Koordinations- und Vollzugsaufgaben zum Bundesgesetz über die Raumplanung. In: Beih. Z. schweiz. Forstver. Nr. 55.

BOSSHARD, W. (1975): Forstliche Gesichspunkte zur Nutzung von Standorten im landwirtschaftlichen Grenzertragsbereich. In: Technische und wirtschaftliche Aspekte der Nutzung von Böden im Grenzertrags-Bereich. Eidg. Anst. forstl. Versuchswes. Ber. 141: S. 28-33.

DUBS, H. (1974): Rechtsfragen der Waldrodung in der Praxis des Bundesgerichts. In: Schweiz. Z. f. Forstw. 125, 5: 275-291.

Eidg. Oberforstinspektorat (1975): Gesamtkonzeption für eine schweizerische Wald- und Holzwirtschaftspolitik. Hauptbericht der vom Eidg. Departement des Innern am 16.8.1971 eingesetzten Expertenkommission, Bern, $417 \mathrm{~S}$.
It is not the purpose of the forest conservation laws to prohibit land-owners from keeping their land free of forest vegetation. If, however, a land-owner does not take steps to prevent the natural growth of such vegetation on unutilized land, what is legally defined as forest may develop. This is particularly the case in the Canton of Tessin, where the favourable climate promotes the rapid growth of forest.

From the forester's viewpoint, future research on unutilized land should focus on the development of feasible methods for the detailed determination and regular inventory of forested areas. Furthermore, in order to include new forest areas in reforestation plans, both suitable criteria should be evolved to identify forest functions and appropriate mapping techniques. Above all, however, future research work should be based on a comprehensive approach incorporating all aspects of the problem.

SCHERRER, H.U., SURBER, E. (1978): Behandlung von Brachland in der Schweiz. Eidg. Anst. forstl. Versuchswes. Ber. 189: $122 \mathrm{~S}$.

SURBER, E., AMIET, R., KOBERT, H. (1973): Das Brachlandproblem der Schweiz. Eidg. Anst. forstl. Versuchswes., Ber. 112: $138 \mathrm{~S}$.

WICKI, C., WEBER, H. (1980): Die Bearbeitung von Problemen der Wald- und Holzwirtschaft sowie des Landschaftsschutzes in Regionalen Entwicklungskonzepten für das Berggebiet. Die Region, Nr. 1180, BIGA, Zentralstelle für regionale Wirtschaftsförderung, S. 12-23. 